\title{
Data-driven vibration-based bearing fault diagnosis using non-steady-state training data
}

\author{
Kurt Pichler $^{1}$, Ted Ooijevaar ${ }^{2}$, Clemens Hesch ${ }^{1}$, Christian Kastl ${ }^{1}$, and Florian Hammer ${ }^{1}$ \\ ${ }^{1}$ Linz Center of Mechatronics GmbH, Altenberger Straße 69, 4040 Linz, Austria \\ ${ }^{2}$ DecisionS, Flanders Make, Gaston Geenslaan 8, 3001 Leuven, Belgium \\ Correspondence: Kurt Pichler (kurt.pichler@1cm.at)
}

Received: 20 September 2019 - Revised: 31 March 2020 - Accepted: 9 April 2020 - Published: 12 May 2020

\begin{abstract}
This paper presents the extension of an empirical study in which a universally applicable fault diagnosis method is used to analyse vibration data of bearings measured with accelerometers. The motivation for extending the previously published results was to provide a profound analysis of the proposed approach with regard to a more feasible training scenario for real applications. For a detailed assessment of the method, data were acquired on two different test beds: a gearbox test bed equipped with various bearings at different health states and an accelerated lifetime (ALT) test bed to degrade a bearing and introduce an operational fault. Features were extracted from the raw data of two different accelerometers and used to monitor the actual health state of the bearings. For that purpose, feature selection and classifier training are performed in a supervised-learning approach. The accuracy is estimated using an independent test dataset. The results of the gearbox test bed data show that the training of the method can be performed with non-steady-state data and that the same feature set can be used for different revolution speeds if a small decrease in accuracy is acceptable. The results of the ALT test bed show that the same features that were identified in the gearbox test start to change significantly when the bearing starts to degrade. Thus, it is possible to observe the identified features for applying predictive maintenance.
\end{abstract}

\section{Introduction}

Manufacturing companies continuously try to increase their productivity, by avoiding machine downtime among other things. The former involves considerable costs because of the resulting loss of turnover. Monitoring the condition of, for instance, bearings and gears plays a vital role in the maintenance programme of rotating machines. Early fault detection could allow for moving from a time-based preventivemaintenance programme to a condition-based predictivemaintenance strategy and reducing unexpected machine downtime and cost.

Vibration-based condition monitoring is an established approach that has been employed by industries for many years in their maintenance programmes (Randall, 2011). However, up to this day, machine operators often still base their maintenance decisions on data from the periodical and manual inspection of single machines, which does not always result in correct conclusions. The common practice is that vibra- tion measurements are periodically recorded using portable vibration sensors, and measurement signals are analysed by an expert to interpret the machine's health condition. This approach can, however, lead to serious misinterpretation, where rapidly growing impairments could be missed.

A continuous condition-monitoring approach enables early detection of machine faults. In this way, the machine condition is continuously tracked, and total failures can be anticipated in advance, hence allowing appropriate maintenance actions. Despite their advantages, continuous monitoring programmes are still not well adopted by industry. Firstly, this is because it often involves a high investment cost. Although recent advancements in sensor, acquisition and processing hardware have demonstrated cost-effective solutions (Albarbar et al., 2008; Ompusunggu et al., 2018), the economic benefit of the investment is still not clear and hard to quantify. Secondly, this is because many of those systems still require an expert to interpret the analysis results. Finally, this is also because it is not straightforward to select the most 
appropriate condition-monitoring method for a specific application.

A wide range of vibration-based bearing fault detection methods have been proposed in the literature (Henriquez et al., 2014; Sait and Sharaf-Eldeen, 2011; Wang et al., 2017; Zarei et al., 2014). Approaches that utilize time domain features (e.g. crest factor and kurtosis; Barbini et al., 2017), frequency and cepstral-domain features (e.g. envelope analysis and cepstral coefficients; Borghesani et al., 2013) usually assume stationary machine conditions. Other methods such as cyclostationary analysis (i.e. second-order technique in the frequency domain; Dalpiaz et al., 2013; Hu et al., 2019) and time-frequency domain analysis (e.g. Wigner-Ville distribution, Hilbert-Huang transform and wavelet-transform-based features; Bajric et al., 2016) are more appropriate for nonstationary processes. Some of those methods are purely data driven, whereas others use the physical relation between the bearing geometry, the rotational shaft speed and the bearingspecific fault frequencies associated to the impulse behaviour introduced by bearing faults.

In this paper, we present a purely data-driven method that extracts a large number of features from vibration data of accelerometer measurements and selects and classifies these features in a supervised-learning approach. Training and test data were acquired at two different bearing test beds: a gearbox setup that can be equipped with bearings of different degradation statuses and a simple rotating shaft with a bearing under certain radial loads for accelerated degradation. However, the method does not address one specific application with certain requirements of the application. We applied the same basic idea to very different applications like fault diagnosis in a hydraulic accumulator loading circuit or oscillation detection. In both cases, we obtained satisfying accuracy values. In any case, the limitation of the method is that it requires information-rich training data of the underlying system to select meaningful features and train suitable classifiers. In this context, information-rich means that the training data have to contain the different possible (fault) states of the application as well as different operation modes. There are of course general requirements that hold for fault detection in most applications, such as the desire to obtain a high detection accuracy and the ability to detect faults under all relevant operational conditions. Both of them are tackled implicitly for the specific application of this paper by the main goals that are stated below.

The basics of the proposed method were already compared to two state-of-the-art methods for bearing monitoring in Ooijevaar et al. (2019). It proved that it can compete with the other methods, although those incorporate specific knowledge about the monitored bearing, while the proposed method is purely data driven. Of course, there are also some drawbacks of the proposed method compared to the other state-of-the-art methods, for instance the requirement of sufficient training data for different states and operation modes.
In contrast to Ooijevaar et al. (2019), the goal of this paper is to analyse if a more feasible training scenario is possible. Therefore, it is investigated if it is sufficient to acquire training data with linearly increasing speed instead of many different steady-state speed levels to save time for training data acquisition. It is also investigated if the same feature set can be used for different revolution speeds of the bearing to make it applicable to different speeds without adapting the feature set.

The paper is structured as follows: in Sect. 2, the problem is briefly stated, and the experimental setup is introduced. The classification method is described in detail in Sect. 3, and Sect. 4 provides test results. Finally, Sect. 5 gives the conclusions of the work.

\section{Problem statement and experimental setup}

In this paper, a previously proposed method (Ooijevaar et al., 2019) for bearing fault detection based on vibration measurements is further analysed with regard to a more feasible training scenario for real-world applications. For that purpose, it is firstly investigated if training data can be acquired using measurements with linearly increasing speed instead of acquiring data at many different steady-state speed levels. That saves a significant amount of time for training data acquisition. Secondly, it is investigated if the same feature set can be used for different revolution speeds of the bearing. That makes the approach more universally applicable, since there is no need to adapt the feature set to the revolution speed.

Since the paper deals with applying the proposed method to bearing fault detection, it is of essential importance to know the underlying physical system. Hence, this section provides a detailed explanation of the experiments and the measured data. Two types of experiments have been performed: (i) an accelerated lifetime test (ALT) of a ball bearing on a single-shaft drive train setup and (ii) a test on a more complex gearbox setup including bearings with various faults. In both test setups, the vibrations of the bearing are measured by accelerometers. These vibration data are used to detect the faults in a machine-learning approach. The tests are described in the next two subsections.

\subsection{Accelerated lifetime test}

The accelerated lifetime test allows for creating an operational fault in a bearing. This test differs from other studies on the fact that those are often limited to artificially induced faults. Moreover, the fault evolution and accumulation can be monitored during the accelerated lifetime. The experimental setup used to perform the accelerated lifetime test is shown in Fig. 1. The setup comprises of a single shaft with a test bearing. The shaft is supported with the help of a support bearing on each side. A hydraulic cylinder is used to apply a radial load to the test bearing up to a maximum of $10 \mathrm{kN}$. The test bearing is oil lubricated by an internal oil bath. Two air 


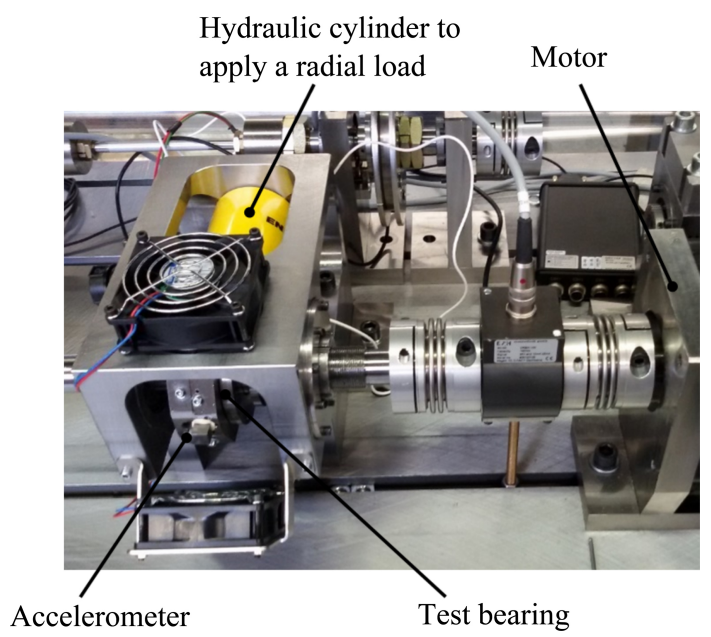

Figure 1. The drive train setup used to reduce the lifetime of a bearing to less than $1 \mathrm{~d}$, allowing for the generation of vibration data during the accumulation of an operational bearing fault.

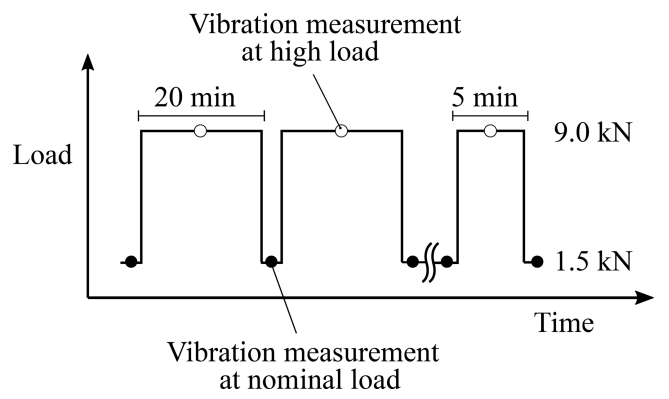

Figure 2. The load was temporarily increased from 1.5 to $9 \mathrm{kN}$ to accelerate the lifetime of the bearing.

fans are installed to cool the setup and avoid overheating of the bearing. The setup is driven by a motor at a fixed rotation speed of $1500 \mathrm{rpm}$.

The test procedure is schematically illustrated in Fig. 2. Vibration measurements were performed under a nominal radial load of $1.5 \mathrm{kN}$ (i.e. $10 \%$ of the dynamic load rating). The radial load was temporarily increased to $9.0 \mathrm{kN}$ (i.e. $65 \%$ of the dynamic load rating) to accelerate the degradation of the bearing. In the beginning, the interval of increased load was $20 \mathrm{~min}$, but this had been reduced as soon as the first indication of an incipient fault was noticed in the measured vibration responses. In total, 30 vibration measurements were performed at the nominal $1.5 \mathrm{kN}$ load condition, and $29 \mathrm{vi}-$ bration measurements were performed at the high $9.0 \mathrm{kN}$ radial load. The accelerated lifetime test was stopped when a vibration peak level of $\pm 50 \mathrm{~g}$ was reached.

The applied radial load, the radial vibrations in the loading direction and the temperature of the bearing housing were measured during the test. The machine vibrations were measured using a piezo-film ACH-01-03 accelerometer and sampled at $12.8 \mathrm{kHz}$ by an embedded acquisition platform. In

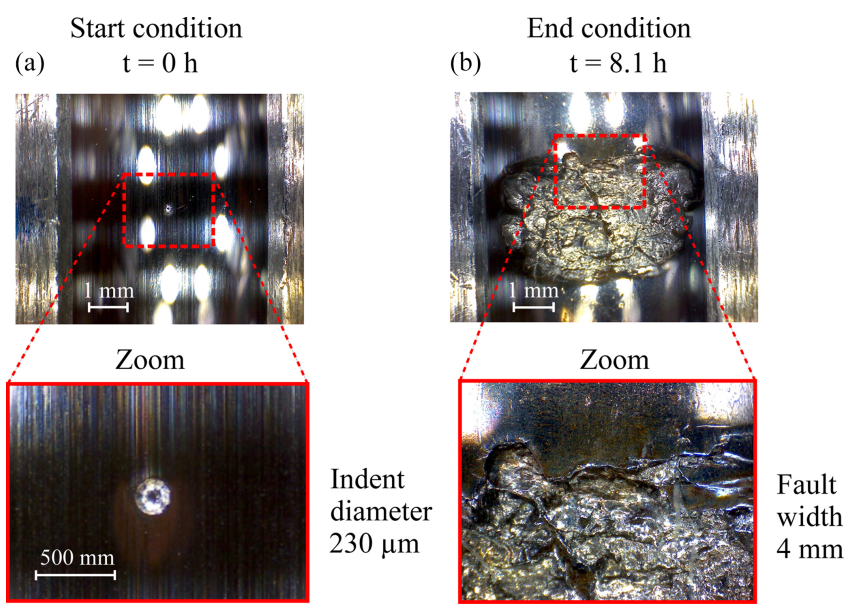

Figure 3. The indentation at the bearing inner race was used as the start condition, and the surface fatigue fault at the inner race was introduced by the accelerated lifetime test.

each measurement, $20 \mathrm{~s}$ of data were acquired. The acquisition platform consists of a BeagleBone Black single-board computer with a Linux operating system, supplemented with a customized six-channel interface. This embedded platform is used as a compact, open, scalable and cost-effective data acquisition system.

The accelerated lifetime test was performed on a FAG 6205 ball bearing. Before the start of the test, a small indentation (see Fig. 3) with a diameter of $230 \mu \mathrm{m}$ was created in the inner race using a Rockwell $\mathrm{C}$ hardness tester. This indentation is used as a local stress riser and represents a local plastic deformation caused by, for instance, a contamination particle. Subsequently, the accelerated lifetime test was performed for several hours. Although bearings can fail in many different ways, the indentation triggers the bearing to fail in a more repeatable way. The test was stopped when severe rolling contact surface fatigue occurred at the inner race (Halme and Andersson, 2009). The start and the end condition of the inner race of the test bearing are shown in Fig. 3.

Only a single dataset has been used in this paper. However, the accelerated lifetime test has been performed several times as part of other research by the authors. They have all resulted in similar surface fatigue faults at the inner race of the test bearing.

\subsection{Gearbox test}

The second test performed in this study was an industrially representative gearbox setup. Figure 4 shows a photograph and a schematic top view of the gearbox setup. The test setup consists of (i) an induction electric motor, (ii) a gearbox and (iii) a magnetic brake. The motor is controlled by a variable-frequency drive (VFD) with either a stationary mode or a transient mode (run-up or run-down mode). The 


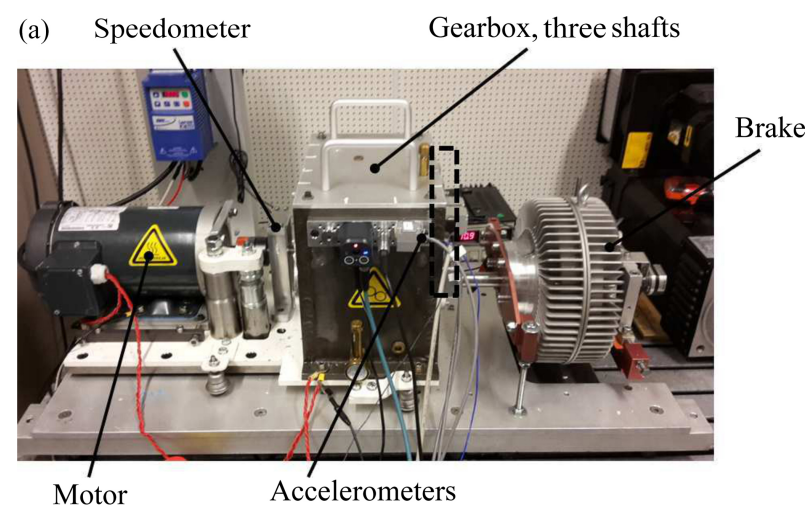

(b)

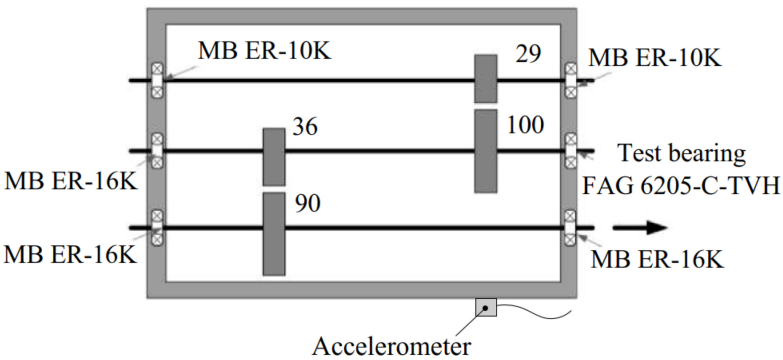

Figure 4. Gearbox setup comprising a motor, three-shaft gearbox and brake to introduce a load.

motor speed can be controlled from 0 to $3000 \mathrm{rpm}$. The gearbox input shaft is connected to the motor through a flexible coupling, while the gearbox output shaft is directly coupled to the brake. The torque applied to the brake can be adjusted by the controller from 0 to $50 \mathrm{Nm}$.

As illustrated in Fig. 4, the gearbox comprises of three parallel shafts connected through contacting spur gear pairs. Note that the number of gear teeth is indicated in the figure. Hence the total reduction factor from the input to the output shaft is equal to $(100 / 29) \times(90 / 36)=8.62$. The input shaft is supported by MB ER-10K deep-groove ball bearings, while the other shafts are supported by MB ER-16K deep-groove ball bearings. For simulating a healthy or faulty state on the gearbox, the right-side bearing housing that supports the second shaft is equipped either with a healthy or a damaged FAG 6205-C-TVH ball bearing.

Two healthy bearings and three faulty bearings with different inner race faults were tested. An indentation fault with a diameter of $490 \mu \mathrm{m}$ was created using an Rockwell C hardness tester. Two other bearings with operational faults were created using the accelerated lifetime test setup as described in Sect. 2.1. The healthy bearings are referred as "Healthy1" and "Healthy2", while the faulty bearings are referred as "Indent", "Faulty1" and "Faulty2", in order of increasing severity; these are illustrated in Fig. 5.

For each healthy or faulty state, two operating conditions were imposed on the gearbox setup, namely two different

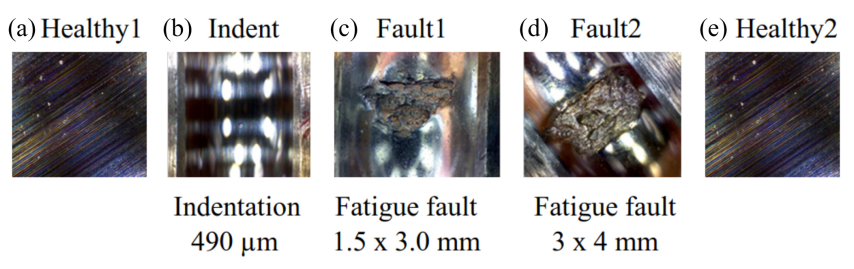

Figure 5. Five bearing states tested on the gearbox setup comprising two healthy bearings and three faulty bearings with different severities.

motor speeds of 1500 and $3000 \mathrm{rpm}$. The brake torque was kept constant at $50 \mathrm{Nm}$. Because of the transmission ratio, the rotational speed of the second shaft is $29 / 100$ lower than that of the motor speed, while the torque applied on the second shaft is $36 / 90$ lower than that of the brake torque. Hence, for the imposed operating conditions, the rotational speeds of the second shaft were 435 and $870 \mathrm{rpm}$, while the torque applied to the second shaft was $20 \mathrm{Nm}$. A high-end PCB (picocoulomb; manufacturer PCB Piezotronics) accelerometer and a low-cost MEMS (microelectromechanical-system) accelerometer were mounted on the gearbox housing as shown in Fig. 4. The vibration signals were sampled at $50 \mathrm{kHz}$ using a Dewesoft data acquisition system. For each operating condition, 10 operations of $20 \mathrm{~s}$ each were repeated. Furthermore, for each of the five tested bearing states, three rampup measurements were conducted. In these ramp-up measurements, the motor speed was increased linearly from 0 to $3000 \mathrm{rpm}$ within $40 \mathrm{~s}$. The ramp-up measurements are used to investigate the first and main aim of this paper, i.e. reducing measurement effort for training data acquisition. It is obvious that conducting $40 \mathrm{~s}$ of ramp-up measurements saves a significant amount of time compared to conducting steadystate measurements for several seconds at many revolution levels. All data are then processed using scripts written in MATLAB.

\section{Classification method}

The fault diagnosis approach presented here is a purely datadriven one; i.e. it incorporates no physical knowledge about the monitored system. This makes it on one hand much more flexible and applicable to many other kinds of systems, machines or components. On the other hand, incorporating extra knowledge usually improves the diagnostic ability of a condition-monitoring system and reduces the necessary amount of training data.

The proposed method applies a supervised-learning approach to annotated measurement data. In the presented application, accelerometers measure vibrations that are caused by the bearing to be monitored. For this purpose, the accelerometer is mounted at the housing of the bearing (see for instance Fig. 1). Since the fault state in the gearbox setup is known, annotated data for classifier training are available. 


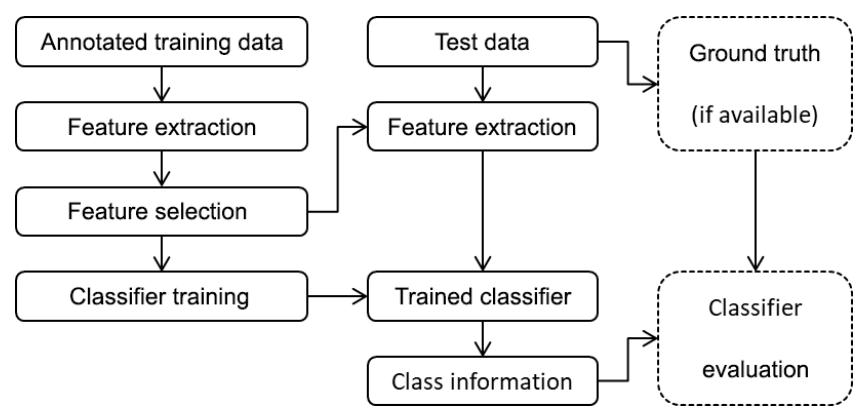

Figure 6. Block diagram of the proposed method.

The training procedure of the proposed method consists of three steps:

- feature extraction from annotated data

- feature selection

- classifier training.

The evaluation procedure for new data consists of two steps:

- extraction of the features selected in the training procedure

- classifier evaluation.

All steps are described in more detail in the following subsections. Moreover, the combination of training and test procedures is summed up in Fig. 6. From the annotated training data, features are extracted, and the most significant features for the classification task are selected. With these features, a classifier is trained. From the test data, the features that were selected before are extracted. The trained classifier is applied to the features to obtain the estimated class information for the test data. If the ground truth of the test data is available, it can be used together with the estimated class information to evaluate the classifier (confusion matrix, accuracy, etc.; de Ridder et al., 2017).

\subsection{Feature extraction}

In the first step, a large number of features is extracted in a sliding-window approach from the raw accelerometer signals. Feature extraction for vibration analysis has been discussed in numerous publications; extensive reviews can be found for instance in Wang et al. (2017) and Singh and Vishwakarma (2015). The extraction of typical statistical features in the time domain is described in Sharma and Parey (2016), Lei et al. (2007), Shen et al. (2013), Decker and Lewicki (2003), Alattas and Basaleem (2007), Boldt et al. (2013), Jalil et al. (2013), Suma and Gurumurthy (2010), and Kollialil et al. (2013). Features in the time-frequency and frequency domains are proposed and investigated in Sharma and Parey (2016), Lei et al. (2007), Alattas and Basaleem (2007), and Boldt et al. (2013). Typical symptom parameters in the frequency domain for rotating machinery are extracted in Wang and Chen (2007). Adopting the spectral kurtosis for vibration monitoring is examined in Rao (2015) and Antoni and Randall (2006). In McClintic et al. (2000) and Assaad et al. (2014), features of residual and difference signals are extracted by using for instance autoregressive models. Features in the wavelet domain are introduced in Heidari Bafroui and Ohadi (2014), Jafarizadeh et al. (2008), Bajric et al. (2016), and Kollialil et al. (2013). Satyam et al. (1994) and Konstantin-Hansen and Herlufsen (2010) examine vibration analysis in the cepstral domain. The application of synchronous time averaging is demonstrated for instance in McFadden and Toozhy (2000). We implemented a broad selection of the proposed features to analyse the measured data. Overall, 83 features were extracted. Amongst the finally selected features of a time series $x=x_{1}, x_{2}, \ldots, x_{n}$ were for instance the root mean square (RMS; Sharma and Parey, 2016) as

$x_{\mathrm{rms}}=\sqrt{\frac{1}{n} \cdot \sum_{i=0}^{n} x_{i}^{2}}$

or the interquartile range (Kollialil et al., 2013) as

$x_{\text {iqa }}=\hat{x}_{0.75}-\hat{x}_{0.25}$,

where

$\hat{x}_{p}= \begin{cases}\frac{1}{2}\left(y_{n \cdot p}+y_{(n \cdot p)+1}\right) & \text { if } n \cdot p \in \mathbb{N} \\ y_{\lfloor n \cdot p+1} & \text { if } n \cdot p \notin \mathbb{N}\end{cases}$

and $y_{1} \leq y_{2} \leq \ldots \leq y_{n}$ are the sorted values of $x$.

Also the symptom parameters (Wang and Chen, 2007) of

$p_{2}=\sqrt{\frac{\sum_{i=1}^{N} f_{i}^{4} \cdot S\left(f_{i}\right)}{\sum_{i=1}^{N} f_{i}^{2} \cdot S\left(f_{i}\right)}}$

and

$p_{4}=\frac{\sigma}{\bar{f}}$,

where $f_{i}, i=1, \ldots, N$ are the frequency bins, $S\left(f_{i}\right)$ is the power spectrum,

$\sigma=\sqrt{\frac{\sum_{i=1}^{N}\left(f_{i}-\bar{f}\right)^{2} \cdot S\left(f_{i}\right)}{N-1}}$

and

$\bar{f}=\frac{\sum_{i=1}^{N} f_{i} \cdot S\left(f_{i}\right)}{\sum_{i=1}^{N} S\left(f_{i}\right)}$,

are amongst the top features. However, for confidentiality reasons we are not allowed to name the exact features that were chosen in each particular test. Therefore, we use abstract feature numbers in the following sections. 


\subsection{Feature selection}

In the next step of the supervised-learning approach, the dimensionality of the feature space is reduced to avoid the curse of dimensionality (Bellman, 2003). Therefore, the significant features are identified by feature selection procedures as described in Guyon and Elisseeff (2003). In particular, a standard forward-selection-filter algorithm selecting one feature per step was applied. As the selection criterion in each step of forward selection, we use the robust Dy-Brodley distance measure (Dy and Brodley, 2004). Assuming a dataset with $C \in \mathbb{N}$ classes in a $k$-dimensional feature space, the feature values for each class can be represented as a matrix of $\mathbf{X}_{c} \in \mathbb{R}^{n_{c} \times k}, c \in\{1, \ldots, C\}$, with $n_{c} \in \mathbb{N}$ denoting the number of samples for class $c$. Then $\boldsymbol{\mu}_{c} \in \mathbb{R}^{k}$ and $\boldsymbol{\Sigma}_{c} \in \mathbb{R}^{k \times k}, c \in\{1, \ldots, C\}$, denote the mean values and covariance matrices of each class $c$, and $\boldsymbol{\mu} \in \mathbb{R}^{k}$ denotes the mean value over all classes. Defining the within-scatter $\mathbf{S}_{\mathrm{W}} \in \mathbb{R}^{k \times k}$ as

$\mathbf{S}_{\mathrm{W}}=\sum_{c=1}^{C} \frac{n_{c}}{n} \cdot \mathbf{\Sigma}_{c}$

and the between scatter $\mathbf{S}_{\mathrm{B}} \in \mathbb{R}^{k \times k}$ as

$\mathbf{S}_{\mathrm{B}}=\sum_{c=1}^{C} \frac{n_{c}}{n} \cdot\left(\boldsymbol{\mu}_{c}-\boldsymbol{\mu}\right)^{\mathrm{T}} \cdot\left(\boldsymbol{\mu}_{c}-\boldsymbol{\mu}\right)$,

where

$n=\sum_{c=1}^{C} n_{c}$,

the Dy-Brodley distance measure is finally defined as

$J=\operatorname{tr}\left(\mathbf{S}_{\mathrm{W}}^{-1} \cdot \mathbf{S}_{\mathrm{B}}\right)$

Feature selection is stopped when the relative gain of the selection criterion falls below $1 \%$. We also performed tests with the Mahalanobis distance (McLachlan, 1999) as the selection criterion; however, both distance measures resulted in the same feature sets.

\subsection{Classifier training}

After feature extraction and selection, a classifier is trained in the feature space. For that purpose, we use linear and quadratic discriminant analysis (Hastie et al., 2009; de Ridder et al., 2017).

In this classification approach, the class conditional distributions of a new data sample $x \in \mathbb{R}^{k}$ are modelled as $P\left(\boldsymbol{x} \mid c=c_{i}\right)$ for each class $c_{i} \in\{1, \ldots, C\}$. By using Bayes' rule of

$$
\begin{aligned}
P\left(c=c_{i} \mid \boldsymbol{x}\right) & =\frac{P\left(\boldsymbol{x} \mid c=c_{i}\right) \cdot P\left(c=c_{i}\right)}{P(\boldsymbol{x})} \\
& =\frac{P\left(\boldsymbol{x} \mid c=c_{i}\right) \cdot P\left(c=c_{i}\right)}{\sum_{c_{j}=1}^{C} P\left(\boldsymbol{x} \mid c=c_{j}\right) \cdot P\left(c=c_{j}\right)}
\end{aligned}
$$

(a) Normal probability plot

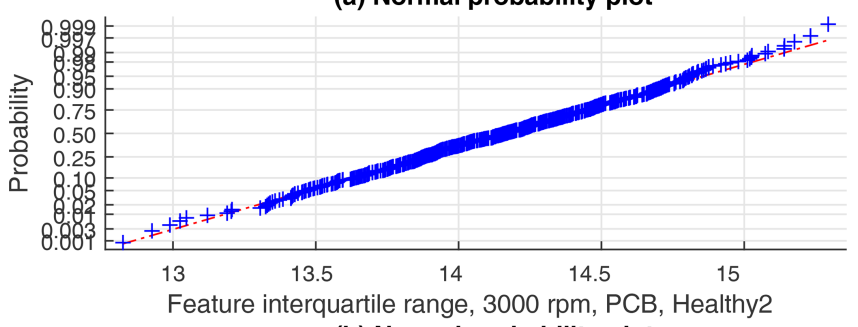

(b) Normal probability plot

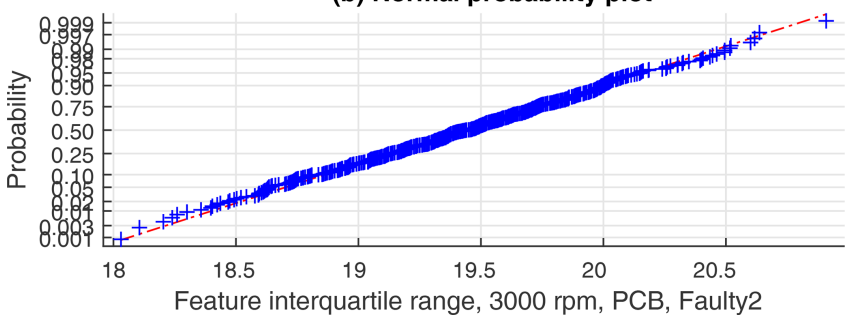

Figure 7. Normal probability plots of the feature interquartile range, $3000 \mathrm{rpm}$ data, PCB sensor, and states Healthy 2 and Faulty2.

and selecting the class $c_{i}$ with highest conditional probability, a class prediction can be made. In the application example of this paper, the classes are "Healthy", "Indent" and "Fault". In discriminant analysis, $P(\boldsymbol{x} \mid c)$ is modelled as a multivariate normal distribution with density

$$
\begin{aligned}
& P\left(\boldsymbol{x} \mid c=c_{i}\right)= \\
& \frac{1}{(2 \pi)^{\frac{k}{2}} \cdot\left|\boldsymbol{\Sigma}_{c_{i}}\right|^{\frac{1}{2}}} \cdot \mathrm{e}^{-\frac{1}{2}\left(\boldsymbol{x}-\boldsymbol{\mu}_{c_{i}}\right)^{\mathrm{T}} \cdot \boldsymbol{\Sigma}_{c_{i}}^{-1} \cdot\left(\boldsymbol{x}-\boldsymbol{\mu}_{c_{i}}\right)},
\end{aligned}
$$

where the prior probabilities $P\left(c=c_{i}\right)$, the class mean values $\boldsymbol{\mu}_{c_{i}}$ and the class covariance matrices $\boldsymbol{\Sigma}_{c_{i}}$ are estimated from training data. Equation (13) represents the quadratic case of discriminant analysis. In the linear case, the normal distributions for each class are assumed to have the same covariance matrix, i.e. $\boldsymbol{\Sigma}_{c_{i}}=\boldsymbol{\Sigma}$ for $c_{i} \in\{1, \ldots, C\}$.

The validity of using normally distributed classifiers was checked by normal probability plots of the feature vectors (see for instance the feature interquartile range for two states in Fig. 7).

The supervised-learning approach implies that the method depends on having a sufficient amount of annotated training data for all states (failure modes) to be monitored. There are also classifiers for one-class classification (also referred to as novelty detection) available (Tax, 2001). However, those techniques detect only a deviation from a nominal state and are thus prone to overdetection due to changing operation modes. Furthermore, the feature selection process depends on having an annotated dataset as well. For the ALT test no training data from different states were available; hence we used a novelty detection technique. For that purpose, the features selected in the gearbox test are observed by cumulativesum (CUSUM) control charts (Hawkins and Olwell, 1998). 
Given annotated training data, the whole process of feature extraction, feature selection and classification can be fully automated. The more useful the information in the training data is, the better the resulting feature subset and classifier are. In this context, information means different states, rotation speed, repeated measurements with different samples of the same bearing type and so on.

\subsection{Evaluation of new data}

The process of evaluating a new data sample is straightforward: the selected features are extracted in a sliding-window approach from the raw accelerometer signals, and the classifier is applied to those features. Since many classifiers are able to deliver class membership probabilities, it is generally also possible to determine instances lying between two distinct states. However, we restrict here the evaluation to crisp class decisions by detecting the maximum class probability for each observation.

If a set of new samples (i.e. the test dataset) contains the true class information (the ground truth), the estimated class can be compared to the ground truth to evaluate the quality of the classifier. For that purpose, many measures like accuracy, balanced accuracy, confusion matrices and receiveroperating-characteristic (ROC) curves are proposed in the literature (de Ridder et al., 2017).

\section{Results}

The results obtained by the proposed method are presented in this section. The accelerated lifetime test results are addressed first. This is followed by the results of the gearbox test.

\subsection{Accelerated lifetime test}

In the ALT test, the features were extracted from the raw accelerometer signals in an overlapping sliding-window approach (window length of $0.2 \mathrm{~s}$ and overlap of $0.1 \mathrm{~s}$ ). That yields 199 observations per $20 \mathrm{~s}$ data batch. However, for final evaluation, only the mean value of those 199 observations of a data batch is observed. Unlike the gearbox setup, we had no data of different health states available for feature selection and classifier training in the accelerated lifetime test. Therefore we could not strictly follow the evaluation scheme proposed in Sect. 3. We were rather restricted to detect significant changes in the feature values. For that purpose, CUSUM control charts were applied to the behaviour of a feature over time. Due to the missing feature selection step, we evaluated the top-ranked features of the gearbox test. All of those features increased significantly towards the end of the test run for the $9.0 \mathrm{kN}$ as well as for the $1.5 \mathrm{kN}$ load conditions. For instance, feature 20 is depicted in Fig. 8. Due to the increasing feature value, the upper threshold of the CUSUM control chart is exceeded after approximately (a) $8 \quad$ Feature: $20,9.0 \mathrm{kN} \quad$ (b)

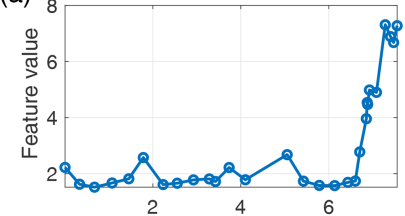

b) $5 \quad$ Feature: $20,1.5 \mathrm{kN}$

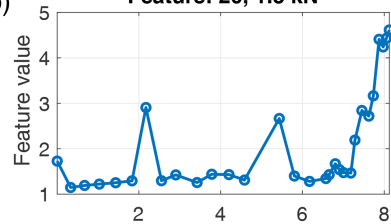

CUSUM control chart
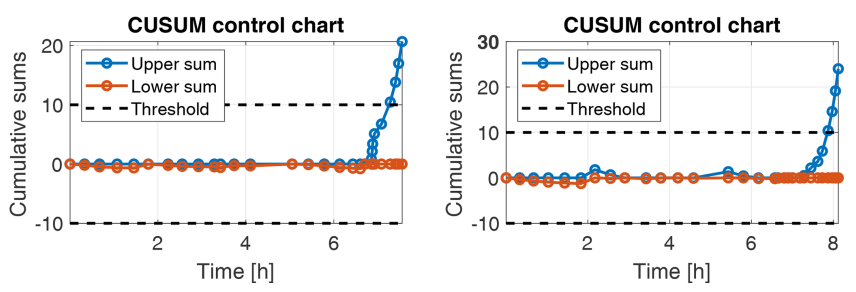

Figure 8. Feature values and CUSUM control charts for feature 20 in the ALT test for load $9.0 \mathrm{kN}$ (a) and $1.5 \mathrm{kN}(\mathbf{b})$.
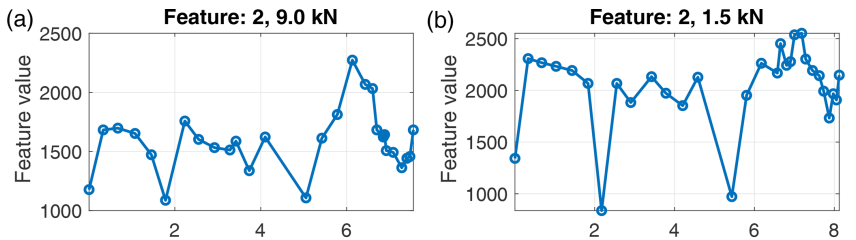

CUSUM control chart
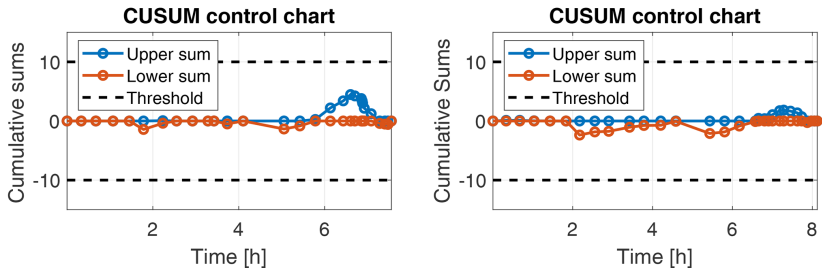

Figure 9. Feature values and CUSUM control charts for feature 2 in the ALT test for load $9.0 \mathrm{kN}$ (a) and $1.5 \mathrm{kN}$ (b).

$7.3 \mathrm{~h}$ in the $9.0 \mathrm{kN}$ case and $7.9 \mathrm{~h}$ in the $1.5 \mathrm{kN}$ case, indicating a failure of the bearing. This result shows that the features identified in the gearbox test can be used to perform predictive maintenance of bearings by observing them using CUSUM control charts. For control purposes, Fig. 9 shows an arbitrarily chosen feature. The feature shows no significant trend, and the CUSUM control charts do not exceed the thresholds. This is a first indication that a wrongly chosen feature will no produce overdetections.

\subsection{Gearbox test}

Just like in the ALT, the features for the gearbox test were extracted from the raw accelerometer signals in an overlapping sliding-window approach with a window length of $0.2 \mathrm{~s}$ and an overlap of $0.1 \mathrm{~s}$, delivering again 199 observations for each $20 \mathrm{~s}$ data batch. After the extraction of all features, the feature selection algorithm was applied to the 3000 and $1500 \mathrm{rpm}$ motor speed data independently. For feature selection, we did not use all available states. We used only the datasets of the states Healthy1, Healthy2 and Faulty2, 


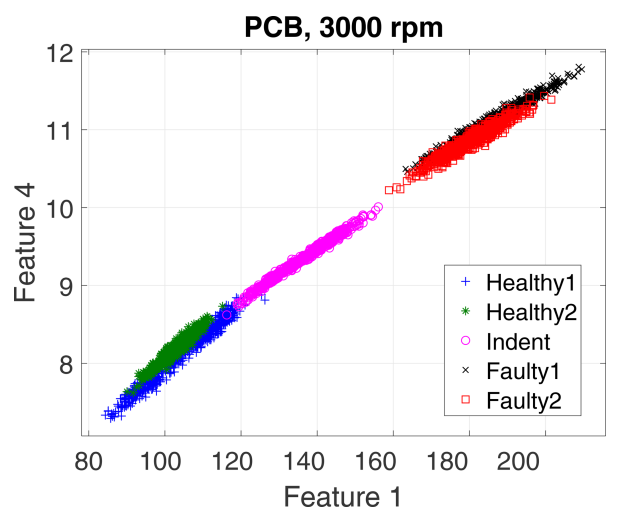

Figure 10. Scatterplot of top two features for the $3000 \mathrm{rpm}$ PCB dataset.

but we did not use Indent and Faulty1. This procedure tests whether the method selects features that are suitable for the other two states as well or not. After feature selection, a quadratic-discriminant-analysis classifier, as described in Sect. 3.3, is trained in the space of the selected and annotated features. Only observations from the states Healthy2, Indent and Faulty 2 are used for classifier training to test the generalizability of the proposed method. Moreover, for feature selection and classifier training, just two arbitrarily chosen data batches from each of the three states are used. That means that $199 \cdot 2 \cdot 3=1194$ observations were available for training. After training, validation was performed with another arbitrarily chosen $20 \mathrm{~s}$ data batch from all five bearing states, hence, $199 \cdot 1 \cdot 5=995$ observations. However, as the target class of the classifier we did not use those five states, but we only used the simplified states Healthy (containing Healthy 1 and Healthy2), Indent and Faulty (containing Faulty 1 and Faulty2). Since the states Healthy 1 and Healthy 2 and Faulty 1 and Faulty 2 are very similar, there is no reason to discriminate between them; in fact they are even supposed to produce similar feature values. As evaluation criteria, we use classification accuracy and the confusion matrix.

First, we evaluate the data of the high-end PCB accelerometer. For the $3000 \mathrm{rpm}$ motor speed data, the algorithm selected three top-ranked features, and for the $1500 \mathrm{rpm}$ data it selected four top-ranked features. However, for a first visual impression we show only the top two features for all recorded states and rotation speeds in a scatterplot in Fig. 10 (3000 rpm) and Fig. 11 (1500 rpm).

The scatterplots already indicate a few possible conclusions:

- Different top features were selected for the different rotation speeds.

- The $3000 \mathrm{rpm}$ dataset revealed better separability.

- Faulty 1 and Faulty 2 produced similar feature values.

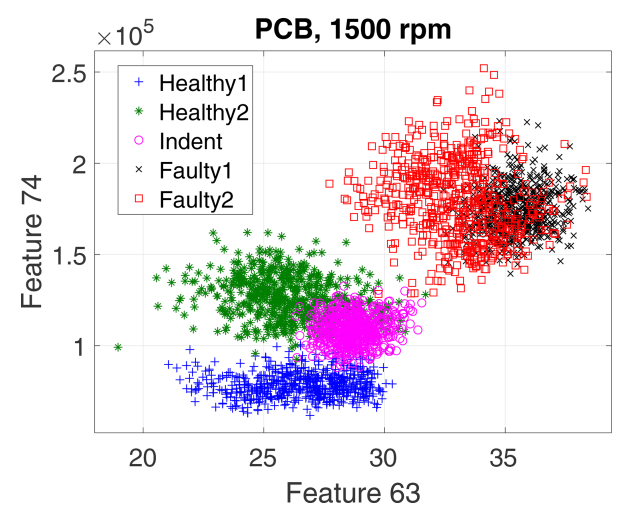

Figure 11. Scatterplot of top two features for the $1500 \mathrm{rpm}$ PCB dataset.

Table 1. Confusion matrix for $3000 \mathrm{rpm}$ PCB data and the top three features.

\begin{tabular}{llrrr}
\hline & & \multicolumn{3}{c}{ Estimated state } \\
\cline { 3 - 5 } & & Healthy & Indent & Faulty \\
\hline \multirow{5}{*}{ True state } & Healthy1 & 192 & 7 & 0 \\
& Healthy2 & 199 & 0 & 0 \\
& Indent & 0 & 199 & 0 \\
& Faulty1 & 0 & 0 & 199 \\
& Faulty2 & 0 & 0 & 199 \\
\hline
\end{tabular}

- In the $3000 \mathrm{rpm}$ dataset, the Indent class lies somewhere in between the healthy and the faulty states.

According to the feature selection step, the $3000 \mathrm{rpm}$ data were validated with the top three features, and the $1500 \mathrm{rpm}$ data were validated with the top four features. The validation yields $99.30 \%$ accuracy for the $3000 \mathrm{rpm}$ data (confusion matrix in Table 1) and $82.41 \%$ accuracy (confusion matrix in Table 2) for the $1500 \mathrm{rpm}$ data. That result confirms the first conclusion above: the separability of the states Healthy, Indent and Faulty in the 3000 rpm case is satisfying, while it is worse in the $1500 \mathrm{rpm}$ case. Especially the state Healthy 1 is misclassified in the $1500 \mathrm{rpm}$ case. Since Healthy1 was not used for classifier training, it is obviously more likely to be misclassified.

To compare the results of the PCB sensor with the MEMS sensor, we extracted the same features from the raw accelerometer data of the MEMS sensor and validated them in the same way. In the $3000 \mathrm{rpm}$ case that yields $93.67 \%$ accuracy (confusion matrix in Table 3; scatterplot of the top two features in Fig. 12), and in the $1500 \mathrm{rpm}$ case that yields $78.99 \%$ accuracy (confusion matrix in Table 4; the scatterplot of the top two features in Fig. 13). In both cases, the accuracy of the low-cost MEMS sensor data is lower than the accuracy of the high-end PCB sensor data. However, one might argue that the comparison is not fair, since the feature selection was performed with the PCB data. In our experi- 
Table 2. Confusion matrix for $1500 \mathrm{rpm}$ PCB data and the top four features.

\begin{tabular}{llrrr}
\hline & & \multicolumn{3}{c}{ Estimated state } \\
\cline { 3 - 5 } & & Healthy & Indent & Faulty \\
\hline \multirow{5}{*}{ True state } & Healthy1 & 33 & 166 & 0 \\
& Healthy2 & 198 & 1 & 0 \\
& Indent & 5 & 194 & 0 \\
& Faulty1 & 0 & 0 & 199 \\
& Faulty2 & 3 & 0 & 196 \\
\hline
\end{tabular}

Table 3. Confusion matrix for $3000 \mathrm{rpm}$ MEMS data and the top three features.

\begin{tabular}{llrrr}
\hline & & \multicolumn{3}{c}{ Estimated state } \\
\cline { 3 - 5 } & & Healthy & Indent & Faulty \\
\hline \multirow{5}{*}{ True state } & Healthy1 & 199 & 0 & 0 \\
& Healthy2 & 138 & 61 & 0 \\
& Indent & 2 & 197 & 0 \\
& Faulty1 & 0 & 0 & 199 \\
& Faulty2 & 0 & 0 & 199 \\
\hline
\end{tabular}

Table 4. Confusion matrix for $1500 \mathrm{rpm}$ MEMS data and the top four features.

\begin{tabular}{llrrr}
\hline & & \multicolumn{3}{c}{ Estimated state } \\
\cline { 3 - 5 } & & Healthy & Indent & Faulty \\
\hline \multirow{5}{*}{ True state } & Healthy1 & 22 & 177 & 0 \\
& Healthy2 & 199 & 0 & 0 \\
& Indent & 8 & 185 & 6 \\
& Faulty1 & 0 & 0 & 199 \\
& Faulty2 & 11 & 7 & 181 \\
\hline
\end{tabular}

ments, the MEMS sensor did not perform significantly better when the feature selection was performed using the MEMS data.

Since the effort for making steady-state speed measurements at every possible speed level is too high, it is desirable to have a less costly training data acquisition procedure. Therefore, we also tested the feasibility of using the ramp-up measurements for training a classifier. For that purpose, the target speed $v_{\mathrm{t}} \mathrm{rpm}$ of the steady-state validation data is used to select a subset of the ramp-up data for classifier training. More specifically, we use the ramp-up data that represent a motor speed of $v=v_{\mathrm{t}} \pm 20 \mathrm{rpm}$ for training. With that data, the classifier is trained and applied to the steady-state validation data in the same way as described above. The accuracy values can be seen in Table 5, and the according confusion matrices are in Table 6 (3000 rpm PCB), Table 7 (3000 rpm MEMS), Table 8 (1500 rpm PCB) and Table 9 (1500 rpm MEMS). We see again that the high-end PCB sensor delivers better accuracy than the low-cost MEMS sensor. Moreover,

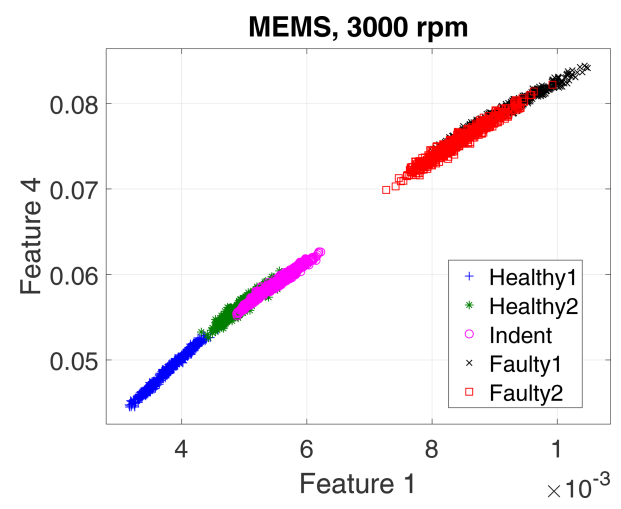

Figure 12. Scatterplot of top two features for the $3000 \mathrm{rpm}$ MEMS dataset.

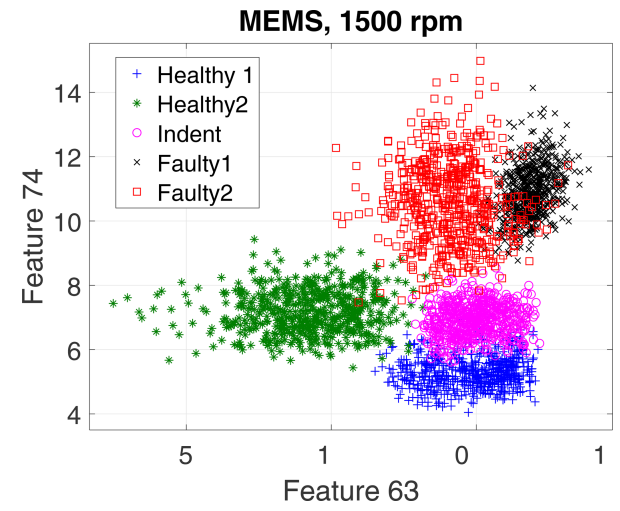

Figure 13. Scatterplot of top two features for the $1500 \mathrm{rpm}$ MEMS dataset.

a higher motor speed allows for more accurate classification than a lower motor speed. However, all accuracy values are too low for a reliable condition-monitoring system.

Interestingly, the accuracy increases significantly when an offset $\Theta$ is added to each dimension of the normalized feature space (normalizing each dimension of the feature space to $\mu=0$ and $\sigma=1$ is a standard procedure in pattern recognition). For the PCB sensor data, an offset value of $\Theta_{\mathrm{PCB}}=$ -0.25 is the best choice, while for the MEMS sensor data, an offset value of $\Theta_{\text {MEMS }}=-0.75$ delivers the best results. The related accuracy values with the additional offsets can be seen in Table 10; the confusion matrices are found in Table 11 (3000 rpm PCB), Table 12 (3000 rpm MEMS data), Table 13 (1500 rpm PCB) and Table 14 (1500 rpm MEMS). In all cases, the accuracy increases by adding the offset to the normalized feature space. This fact might be due to dynamic effects of the ramp-up measurements; however, the exact reason will be the subject of further investigations of the data in the future. In this test, the same offset was added to all dimensions of the feature space. An individual offset for each dimension might further increase the accuracy. The offset was determined by a simple grid search approach using accuracy as the target value. For future applications it is de- 
Table 5. Accuracy values for classifier training with ramp-up data.

\begin{tabular}{cccc}
\hline & \multicolumn{2}{c}{ Accelerometer } \\
\cline { 3 - 4 } & & PCB & MEMS \\
\hline \multirow{2}{*}{ Motor speed } & 3000 & $83.12 \%$ & $80.50 \%$ \\
& 1500 & $66.73 \%$ & $56.99 \%$ \\
\hline
\end{tabular}

Table 6. Confusion matrix for $3000 \mathrm{rpm}$ PCB data and the top three features, classifier trained with ramp-up data.

\begin{tabular}{llrrr}
\hline & & \multicolumn{3}{c}{ Estimated state } \\
\cline { 3 - 5 } & & Healthy & Indent & Faulty \\
\hline \multirow{5}{*}{ True state } & Healthy1 & 100 & 99 & 0 \\
& Healthy2 & 133 & 66 & 0 \\
& Indent & 0 & 196 & 3 \\
& Faulty1 & 0 & 0 & 199 \\
& Faulty2 & 0 & 0 & 199 \\
\hline
\end{tabular}

Table 7. Confusion matrix for $3000 \mathrm{rpm}$ MEMS data and the top four features, classifier trained with ramp-up data.

\begin{tabular}{llrrr}
\hline & & \multicolumn{3}{c}{ Estimated state } \\
\cline { 3 - 5 } & & Healthy & Indent & Faulty \\
\hline \multirow{5}{*}{ True state } & Healthy1 & 183 & 16 & 0 \\
& Healthy2 & 197 & 2 & 0 \\
& Indent & 0 & 23 & 176 \\
& Faulty1 & 0 & 0 & 199 \\
& Faulty2 & 0 & 0 & 199 \\
\hline
\end{tabular}

sirable to find a way for a more sophisticated determination of an optimal offset.

As stated before, feature selection was performed for the $3000 \mathrm{rpm}$ data and the $1500 \mathrm{rpm}$ data separately. However, as stated in Sect. 1, it is desirable that the same feature set can be used for different revolution speeds to make the approach more universally applicable. Hence we also applied the features of the $3000 \mathrm{rpm}$ data to the $1500 \mathrm{rpm}$ data to assess the generalizability of those features. The resulting accuracy values for the test runs with steady-state and with ramp-up training data are shown in Table 15. In most cases (except the $1500 \mathrm{rpm}$ MEMS data in the case of ramp-up training), the accuracy using the optimal feature set for $1500 \mathrm{rpm}$ and the tested $3000 \mathrm{rpm}$ feature set is rather similar. That implies that the $3000 \mathrm{rpm}$ feature set is basically applicable to both cases. However, the accuracy values for the $1500 \mathrm{rpm}$ case are too low for reliable bearing fault detection.

\section{Conclusions}

In this paper, we evaluate a previously proposed approach for the data-driven fault detection of bearings with regard to
Table 8. Confusion matrix for $1500 \mathrm{rpm}$ PCB data and the top three features, classifier trained with ramp-up data.

\begin{tabular}{llrrr}
\hline & \multicolumn{3}{c}{ Estimated state } \\
\cline { 3 - 5 } & & Healthy & Indent & Faulty \\
\hline \multirow{5}{*}{ True state } & Healthy1 & 8 & 191 & 0 \\
& Healthy2 & 186 & 4 & 9 \\
& Indent & 127 & 72 & 0 \\
& Faulty1 & 0 & 0 & 199 \\
& Faulty2 & 0 & 0 & 199 \\
\hline
\end{tabular}

Table 9. Confusion matrix for $1500 \mathrm{rpm}$ MEMS data and the top four features, classifier trained with ramp-up data.

\begin{tabular}{llrrr}
\hline & & \multicolumn{3}{c}{ Estimated state } \\
\cline { 3 - 5 } & & Healthy & Indent & Faulty \\
\hline \multirow{5}{*}{ True state } & Healthy1 & 4 & 125 & 70 \\
& Healthy2 & 197 & 2 & 0 \\
& Indent & 0 & 154 & 45 \\
& Faulty1 & 0 & 1 & 198 \\
& Faulty2 & 155 & 30 & 14 \\
\hline
\end{tabular}

Table 10. Accuracy values for classifier training with ramp-up data and additional offset.

\begin{tabular}{cccc}
\hline & \multicolumn{2}{c}{ Accelerometer } \\
\cline { 3 - 4 } & & PCB & MEMS \\
\hline \multirow{2}{*}{ Motor speed } & 3000 & $96.99 \%$ & $92.26 \%$ \\
& 1500 & $75.88 \%$ & $58.69 \%$ \\
\hline
\end{tabular}

Table 11. Confusion matrix for $3000 \mathrm{rpm}$ PCB data and the top three features, classifier trained with ramp-up data, with an additional offset in the steady-state validation data.

\begin{tabular}{llrrr}
\hline & \multicolumn{3}{c}{ Estimated state } \\
\cline { 3 - 5 } & & Healthy & Indent & Faulty \\
\hline \multirow{5}{*}{ True state } & Healthy1 & 179 & 20 & 0 \\
& Healthy2 & 189 & 10 & 0 \\
& Indent & 0 & 199 & 0 \\
& Faulty1 & 0 & 0 & 199 \\
& Faulty2 & 0 & 0 & 199 \\
\hline
\end{tabular}

two main questions. Firstly, is it possible to train the proposed classifier even with non-steady-state measurements? Secondly, can the same feature sets be applied to different revolution speeds of the bearings? For that purpose, appropriate data have been acquired on a test bench and used for evaluation.

The results of this empirical study show that it is feasible to train the bearing fault diagnosis method with ramp-up 
Table 12. Confusion matrix for $3000 \mathrm{rpm}$ MEMS data and the top four features, classifier trained with ramp-up data, with an additional offset in the steady-state validation data.

\begin{tabular}{llrrr}
\hline & & \multicolumn{3}{c}{ Estimated state } \\
\cline { 3 - 5 } & & Healthy & Indent & Faulty \\
\hline \multirow{5}{*}{ True state } & Healthy1 & 197 & 1 & 1 \\
& Healthy2 & 193 & 6 & 0 \\
& Indent & 0 & 130 & 69 \\
& Faulty1 & 0 & 0 & 199 \\
& Faulty2 & 0 & 0 & 199 \\
\hline
\end{tabular}

Table 13. Confusion matrix for $1500 \mathrm{rpm}$ PCB data and the top three features, classifier trained with ramp-up data, with an additional offset in the steady-state validation data.

\begin{tabular}{llrrr}
\hline & & \multicolumn{3}{c}{ Estimated state } \\
\cline { 3 - 5 } & & Healthy & Indent & Faulty \\
\hline \multirow{5}{*}{ True state } & Healthy1 & 54 & 145 & 0 \\
& Healthy2 & 185 & 8 & 6 \\
& Indent & 81 & 118 & 0 \\
& Faulty1 & 0 & 0 & 199 \\
& Faulty2 & 0 & 0 & 199 \\
\hline
\end{tabular}

data if the additional constant is added to the normalized feature space. The accuracy decrease is acceptable compared to steady-state training data, except for the case of the MEMS sensor and low revolution speed of $1500 \mathrm{rpm}$. A similar conclusion can be drawn regarding the other main goal of the paper. The $3000 \mathrm{rpm}$ feature set performs acceptable even on the $1500 \mathrm{rpm}$ data (compared to the $1500 \mathrm{rpm}$ feature set). Again, the only exception is the $1500 \mathrm{rpm}$ ramp-up data with the MEMS sensor, which delivers just the baseline accuracy. Overall, the accuracy of the $1500 \mathrm{rpm}$ test is too low for a reliable fault diagnosis system.

Furthermore, it can be concluded that the classification accuracy increases with the higher rotational speed of the bearing, and the high-end PCB sensor allows for higher accuracy values than the low-cost MEMS sensor. Hence it is not possible to save costs by using less expensive sensors without accepting a ]significant decrease of detection accuracy.

The results of the ALT test show that an upcoming fault can be detected considerably before total failure of the bearing occurs. This leads to the conclusion that observing the selected features with CUSUM control charts is applicable for performing the predictive maintenance of bearings. In addition, a wrongly chosen feature does not lead to overdetections.

However, even though the results are promising, there are still investigations to be made. As mentioned before, the classifier can be trained with dynamic ramp-up data, and an additional offset in the normalized feature space allows for clas-
Table 14. Confusion matrix for $1500 \mathrm{rpm}$ MEMS data and the top four features, classifier trained with ramp-up data, with an additional offset in the steady-state validation data.

\begin{tabular}{llrrr}
\hline & & \multicolumn{3}{c}{ Estimated state } \\
\cline { 3 - 5 } & & Healthy & Indent & Faulty \\
\hline \multirow{5}{*}{ True state } & Healthy1 & 44 & 141 & 14 \\
& Healthy2 & 198 & 1 & 0 \\
& Indent & 4 & 174 & 21 \\
& Faulty1 & 21 & 14 & 164 \\
& Faulty2 & 167 & 28 & 4 \\
\hline
\end{tabular}

Table 15. Accuracy values for using the $3000 \mathrm{rpm}$ feature set for the $1500 \mathrm{rpm}$ data.

\begin{tabular}{llcc}
\hline \multirow{2}{*}{ Training data } & \multicolumn{2}{c}{ Accelerometer } \\
\cline { 3 - 4 } & & PCB & MEMS \\
\hline \multirow{2}{*}{ Steady-state data } & $79.80 \%$ & $78.49 \%$ \\
& Ramp-up data (+ offset) & $72.46 \%$ & $40.00 \%$ \\
\hline
\end{tabular}

sification results that are almost as good as training with steady-state data. The exact reason for that offset will be topic of future analysis of the acquired data. The offset in the feature space was determined in a simple grid search approach. A more sophisticated choice of an optimal offset is desirable for the future. Moreover, we tested just two steadystate motor speeds. Further tests with more speeds will help to evaluate down to what speed the method delivers satisfying results and which features are best suited for different revolution speeds.

Data availability. The datasets that we used cannot made publicly available on a public repository. However, we do offer condition-monitoring datasets to interested parties. The following web page gives an overview of the available datasets: https: //www.flandersmake.be/en/dataset-condition-monitoring (last access: 11 May 2020). Both of the setups that are used in the paper (i.e. the gearbox and ALT setup) are listed on this page. A free sample is provided, but upon request a full dataset can be purchased for e.g. algorithm development, testing or validation purposes. A similar web page is https://www.flandersmake.be/en/datasets (last access: 11 May 2020).

Author contributions. KP applied the proposed method to the acquired data, evaluated the results and prepared the paper with contributions from all co-authors. TO designed the experiments, carried them out and gave valuable input for processing the measured data. Moreover, he wrote significant parts of the Introduction and the Problem statement and experimental setup sections. $\mathrm{CH}$ administered the project and reviewed the paper carefully. CK supervised the research activity and the paper-writing process. FH carefully 
proofreading the paper and made significant changes to improve clarity.

Competing interests. The authors declare that they have no conflict of interest.

Special issue statement. This article is part of the special issue "Sensors and Measurement Systems 2019". It is a result of the "Sensoren und Messsysteme 2019, 20. ITG-/GMA-Fachtagung", Nuremberg , Germany, 25-26 June 2019.

Acknowledgements. The work described in this paper was performed as part of the Mechatronics Alliance, an alliance consisting of the Linz Center of Mechatronics (Austria), Flanders Make (Belgium), FIMECC (Finland) and the E-TIME Institute (France).

Financial support. This research has been supported by the COMET-K2 Center of the Linz Center of Mechatronics (LCM), which is funded by the Austrian federal government and the federal state of Upper Austria, and by Flanders Make, the strategic research centre for the manufacturing industry.

Review statement. This paper was edited by Stefan Rupitsch and reviewed by one anonymous referee.

\section{References}

Alattas, M. and Basaleem, M.: Statistical Analysis of Vibration Signals for Monitoring Gear Condition, Damascus Univ. Journal, 23, 67-92, 2007.

Albarbar, A., Mekid, S., Starr, A., and Pietruszkiewicz, R.: Suitability of MEMS accelerometers for condition monitoring: An experimental study, Sensors, 8, 784-799, 2008.

Antoni, J. and Randall, R.: The spectral kurtosis: application to the vibratory surveillance and diagnostics of rotating machines, Mech. Syst. Signal Pr., 20, 308-331, 2006.

Assaad, B., Eltabach, M., and Antoni, J.: Vibration based condition monitoring of a multistage epicyclic gearbox in lifting cranes, Mech. Syst. Signal Pr., 42, 351-367, 2014.

Bajric, R., Zuber, N., Skrimpas, G., and Mijatovic, N.: Feature Extraction Using Discrete Wavelet Transform for Gear Fault Diagnosis of Wind Turbine Gearbox, Shock and Vibration, 2016, 6748469, https://doi.org/10.1155/2016/6748469, 2016.

Barbini, L., Ompusunggu, A., Hillis, A., du Bois, J., and Bartic, A.: Phase editing as a signal pre-processing step for automated bearing fault detection, Mech. Syst. Signal Pr., 91, 407-421, 2017.

Bellman, R.: Dynamic programming, Courier Dover Publications, Mineola, USA, 2003.

Boldt, F., Rauber, T., and Varejão, F.: Feature Extraction and Selection for Automatic Fault Diagnosis of Rotating Machinery, ENIAC 2013 - X Encontro Nacional de Inteligencia Artificial e Computacional (ENIAC), 19-24 October 2013, Fortaleza, Ceara, Brazil, 2013.
Borghesani, P., Pennacchi, P., Randall, R. B., Sawalhi, N., and Ricci, R.: Application of cepstrum pre-whitening for the diagnosis of bearing faults under variable speed conditions, Mech. Syst. Signal Pr., 36, 370-384, 2013.

Dalpiaz, G., Rubini, R., D’Elia, G., Cocconcelli, M., Chaari, F., Zimroz, R., Bartelmus, W., and Haddar, M., eds.: Advances in Condition Monitoring of Machinery in Non-Stationery Operations, Springer-Verlag Berlin Heidelberg, Germany, 2013.

Decker, H. and Lewicki, D.: Spiral Bevel Pinion Crack Detection in a Helicopter Gearbox, in: Proceedings of the 59th Annual Forum and Technology Display, 6-8 May 2003, Phoenix, Arizona, USA, 2003.

de Ridder, D., Tax, D., Lei, B., Xu, G., Feng, M., Zou, Y., and van der Heijden, F., eds.: Classification, Parameter Estimation and State Estimation: An Engineering Approach Using MATLAB, 2nd Edition, John Wiley \& Sons, Ltd., Chichester, UK, 2017.

Dy, J. and Brodley, C.: Feature Selection for Unsupervised Learning, J. Mach. Learn. Res., 5, 845-889, 2004.

Guyon, I. and Elisseeff, A.: An introduction to variable and feature selection, J. Mach. Learn. Res., 3, 1157-1182, 2003.

Halme, J. and Andersson, P.: Rolling contact fatigue and wear fundamentals for rolling bearing diagnostics - state of the art, Journal of Engineering Tribology, 224, 377-393, 2009.

Hastie, T., Tibshirani, R., and Friedman, J.: The elements of statistical learning: data mining, inference and prediction, Springer Verlag, New York, USA, Berlin, Heidelberg, Germany, 2009.

Hawkins, D. and Olwell, D. (Eds.): Cumulative Sum Charts and Charting for Quality Improvement, Springer-Verlag, New York, USA, 1998.

Heidari Bafroui, H. and Ohadi, A.: Application of wavelet energyband Shannon entropy for feature extraction in gearbox fault detection under varying speed conditions, Neurocomputing, 133, 437-445, 2014.

Henriquez, P., Alonso, J., Ferrer, M., and Travieso, C.: Review of automatic fault diagnosis systems using audio and vibration signals, IEEE T. Syst. Man Cy.-S., 44, 642-652, 2014.

Hu, A., Xiang, L., Xu, S., and Lin, J.: Frequency Loss and Recovery in Rolling Bearing Fault Detection, Chinese Journal of Mechanical Engineering, 32, 35, https://doi.org/10.1186/s10033019-0349-3, 2019.

Jafarizadeh, M., Hassannejad, R., Ettefagh, M., and Chitsaz, S.: Asynchronous input gear damage diagnosis using time averaging and wavelet filtering, Mech. Syst. Signal Pr., 22, 172-201, 2008.

Jalil, M., Butt, F. A., and Malik, A.: Short-time energy, magnitude, zero crossing rate and autocorrelation measurement for discriminating voiced and unvoiced segments of speech signals, in: The International Conference on Technological Advances in Electrical, Electronics and Computer Engineering, 9-11 May 2013, Konya, Turkey, 208-212, 2013.

Kollialil, E. S., Gopan, K. G., Harsha, A., and Joseph, L. A.: Single feature-based non-convulsive epileptic seizure detection using multi-class SVM, in: 2013 International Conference on Emerging Trends in Communication, Control, Signal Processing and Computing Applications (C2SPCA), 10-11 October 2013, Bangalore, India, 1-6, 2013. 
Konstantin-Hansen, H. and Herlufsen, H.: Envelope and Cepstrum Analyses for Machinery Fault Identification, Sound \& Vibration, 44, 10-12, 2010.

Lei, Y., He, Z., Zi, Y., and Hu, Q.: Fault diagnosis of rotating machinery based on multiple ANFIS combination with GAs, Mech. Syst. Signal Pr., 21, 2280-2294, 2007.

McClintic, K., Lebold, M., Maynard, K., Byington, C., and Campbell, R.: Residual and difference feature analysis with transitional gearbox data, in: Proceedings of the 54th Meeting of the Society for Machinery Failure Prevention Technology, 1-4 May 2000, Virginia Beach, Virginia, USA, 2000.

McFadden, P. and Toozhy, M.: Applications of Synchronous Averaging to Vibration Monitoring of Rolling Element Bearings, Mech. Syst. Signal Pr., 14, 891-906, 2000.

McLachlan, G.: Mahalanobis distance, Resonance, 4, 20-26, 1999.

Ompusunggu, A. P., Ooijevaar, T., Kilundu, B., and Devos, S.: Automated bearing fault diagnostics with cost-effective vibration sensor, in: Asset Intelligence through Integration and Interoperability and Contemporary Vibration Engineering Technologies. Proceedings of the 12th World Congress on Engineering Asset Management, 24-26 September 2018, Stavanger, Norway, edited by: Mathew, J., Lim, C., and Ma, L., Springer International Publishing, 463-472, 2018.

Ooijevaar, T., Pichler, K., Di, Y., and Hesch, C.: A Comparison of Vibration based Bearing Fault Diagnostic Methods, International Journal of Prognostics and Health Management, 10, 008, ISSN 2153-2648, 2019.

Randall, R.: Vibration-based Condition Monitoring: Industrial, Aerospace and Automotive Applications, John Wiley \& Sons, Ltd., Chichester, UK, 2011.

Rao, V.: Spectral Kurtosis Theory: A Review through Simulations, Global Journal of Researches in Engineering: Electrical and Electronics Engineering, 15, 49-61, 2015.

Sait, A. and Sharaf-Eldeen, Y.: A Review of Gearbox Condition Monitoring Based on vibration Analysis Techniques Diagnostics and Prognostics, in: Rotating Machinery, Structural Health Monitoring, Shock and Vibration, vol. 5, Proceedings of the 29th IMAC, A Conference on Structural Dynamics, 31 January3 February 2011, Jacksonville, FL, USA, edited by: Proulx, T., 359-374, The Society for Experimental Mechanics, Inc., 2011.
Satyam, M., Sudhakara, R., and Devy, C.: Cepstrum Analysis - An Advanced Technique in Vibration Analysis of Defects in Rotating Machinery, Defence Sci. J., 44, 53-60, 1994.

Sharma, V. and Parey, A.: A review of gear fault diagnosis using various condition indicators, Procedia Engineer., 144, 253-263, 2016.

Shen, C., Wang, D., Kong, F., and Tse, P.: Fault diagnosis of rotating machinery based on the statistical parameters of wavelet packet paving and a generic support vector regressive classifier, Measurement, 46, 1551-1564, 2013.

Singh, S. and Vishwakarma, M.: A Review of Vibration Analysis Techniques for Rotating Machines, International Journal of Engineering Research \& Technology, 4, 757-761, 2015.

Suma, S. A. and Gurumurthy, K. S.: Novel pitch extraction methods using average magnitude difference function (AMDF) for LPC speech coders in noisy environments, in: 2010 2nd International Conference on Signal Processing Systems, 5-7 July 2010, Dalian, China, vol. 1, V1-636-V1-640, 2010.

Tax, D.: One-class classification, $\mathrm{PhD}$ thesis, Delft University of Technology, Delft, the Netherlands, 2001.

Wang, D., Tsui, K., and Mia, Q.: Prognostics and Health Management: A Review of Vibration Based Bearing and Gear Health Indicators, IEEE Access, 6, 665-676, 2017.

Wang, H. and Chen, P.: Fault Diagnosis of Centrifugal Pump Using Symptom Parameters in Frequency Domain, Agricultural Engineering International: the CIGR Ejournal, IX, 1-14, 2007.

Zarei, J., Tajeddini, M., and Karimi, H.: Vibration analysis for bearing fault detection and classification using an intelligent filter, Mechatronics, 24, 151-157, 2014. 